\title{
Computers in biofeedback research and therapy
}

\author{
RONETTE L. KOLOTKIN, KATHERINE A. BILLINGHAM, and HOWARD S. FELDMAN \\ Rush-Presbyterian-St. Luke's Medical Center, Chicago, Illinois 60612
}

\begin{abstract}
While biofeedback is being used extensively in the clinic and the laboratory, a great deal of variability exists among biofeedback users with respect to instrumentation, treatment protocols, techniques of data acquisition, and methods of data analysis. It is argued that the use of computers in biofeedback will help reduce some of this variability. Other advantages of computers in biofeedback are discussed. Several presently available automated systems are reviewed. Implications for future biofeedback research are also discussed.
\end{abstract}

Numerous research and clinical reports document the use of biofeedback in the training of muscle activity (e.g., Budzynski, Stoyva, Adler, \& Mullaney, 1973; Gessel, 1975; Phillips, 1977), brain wave activity (e.g., Nowlis \& Kamiya, 1970; Hart, Note 1), blood pressure (e.g., Blanchard, Young, \& Haynes, 1975; Shapiro, Tursky, \& Schwartz, 1970), heart rate (e.g., Brener, Kleinman, \& Goesling, 1969; Hnatiow \& Lang, 1965), skin surface temperature (e.g., Johnson \& Turin, 1975; Surwit, 1973), and electrodermal activity (e.g., Shapiro, Schwartz, Shnidman, Nelson, \& Silverman, 1972). Responses investigated less frequently include penile erection, mastication, stomach acidity, nasality in speech, salivary flow, respiration, visual accommodation, and anal-sphincter pressure. Biofeedback has also played a major role in the treatment of a variety of disorders, for example, tension headache, migraine, essential hypertension, anxiety, temporomandibular joint syndrome, premature ventricular contractions, asthma, primary dysmenorrhea, functional diarrhea, Raynaud's disease, sinus tachycardia, peptic ulcers, and fecal incontinence (Blanchard \& Epstein, 1978; Silver \& Blanchard, 1978).

Although biofeedback was initially regarded with enthusiasm, recent reviewers of the research and clinical literature have questioned the efficacy of biofeedback techniques. Reviewers have begun to ask whether or not physiological responses can be trained without the use of biofeedback instrumentation (e.g., Alexander \& Smith, 1979; Silver \& Blanchard, 1978). A consistent problem in biofeedback outcome research has been the lack of replicability of findings across studies. While some of this variability in outcome may be related to issues of experimental design, a good portion may be accounted for by differences across studies in instrumentation,

Requests for reprints should be sent to Ronette Kolotkin, Department of Psychology, Rush-Presbyterian-St. Luke's Medical Center, 1753 West Congress Parkway, Chicago, Illinois 60612. biofeedback treatment protocols, techniques of data acquisition, and methods for data analysis.

This paper focuses on these sources of variability and presents an argument in favor of using computers in biofeedback research and clinical work. We also discuss recent research on automated biofeedback systems, as well as the implications of these systems for the fields of behavioral medicine and the experimental analysis of behavior.

A distinction is made here between the "biofeedback system" and the "biofeedback process." While it is possible to automate a biofeedback system (i.e., one can add instrumentation that reduces error and variability, automatically collects, stores, and analyzes data, feeds back processed data to the trainee, and can be programmed to create treatment protocols that are either standardized or self-modifying according to the needs of the trainee), there are several reasons why one cannot automate the biofeedback process. That is, one cannot eliminate the role of "person variables" in the process of doing biofeedback. Briefly, these reasons are as follows: (1) While biofeedback training focuses on changing physiological responses, it is impossible to isolate physiological responses from the rest of a person's life, environment, and psychological makeup. (2) As a result, interacting with the machinery is only part of the process of client change. (3) Individuals react very differently to the experience of biofeedback, depending on their life histories, personalities, and cognitive frameworks. And (4) biofeedback operators may need to be experienced in psychotherapy in order to better help clients to cope with symptoms, to understand the role of the symptoms in their lives, and to become aware of how symptom changes may affect other aspects of their lives. (For further discussion of these issues, see Fuller, 1978; Lazarus, 1977; Price, 1974; Schwartz, 1973). While we acknowledge the importance of person variables in the biofeedback process, discussion of these variables is limited in favor of technological and methodological issues of biofeedback systems. 


\section{SOURCES OF VARIABILITY}

\section{Instrumentation}

A major source of variability in biofeedback research and clinical work results from differences in instrumentation. The earlier devices were especially plagued by poor technical quality and wide variability in functional characteristics. In an examination of 13 of the early EEG feedback devices, Schwitzgebel and Rugh (1975) reported that input impedance ranged from $500 \mathrm{ohms}$ to greater than 2 megohms, alpha filter bandwidths ranged from .9 to $16.5 \mathrm{~Hz}$, and 5 of the 13 devices did not employ differential input amplifiers and were subject to $60-\mathrm{Hz}$ interference.

Although technological advances have helped to improve biofeedback equipment over the years, there is still a large amount of variability in the functional characteristics of the equipment (Rugh, 1979). For example, there is a wide range of bandwidth frequencies currently employed by biofeedback instrument manufacturers. Rugh and Schwitzgebel (1977) ran comparative tests on 11 commercial EMG feedback devices and found that filter bandwidth (which specifies the particular signal frequencies that will be examined) varied from $55 \mathrm{~Hz}$ to $2,600 \mathrm{~Hz}$. To complicate matters, a great majority of biofeedback research and clinical reports do not include adequate information about the filtering characteristics of the apparatus (Ancoli \& Kamiya, Note 2). Unfortunately, different readings are obtained, depending on the bandwidth being used, since the noise level of a device is closely related to filter bandwidth. While narrow-band devices $(100$ to $150 \mathrm{~Hz})$ have very low noise levels, they sample only a small portion of the EMG signal and may not provide an accurate indication of EMG amplitude. On the other hand, wide-band devices $(20-1,000 \mathrm{~Hz})$ have higher noise levels, but they sample more of the EMG signal. According to Peffer (1979), the choice of bandwidth may be less important than the consistency of that choice across and within subjects, especially if different instruments are being used. "There is no simple way available to compensate for measurements made with different bandpass parameters" (Peffer, 1979, p. 261).

Different methods of amplitude quantification also produce different readings. Unfortunately, differences in procedures used exist among biofeedback manufacturers (Rugh \& Schwitzgebel, 1977). At least three methods of amplitude measurement are currently being used in biofeedback work: signal averaging, peak-to-peak measurement, and true root mean squared. A discussion of the mathematical and technical aspects of each of these measures of amplitude quantification may be found in Peffer (1979).

Another difference between various biofeedback devices has to do with the nature of the output signal. Whereas some instruments provide a linear relationship between the input and output signals, others provide the user with a logarithmic transformation (Rugh \&
Schwitzgebel, 1977). Although a logarithmic signal makes changes at the low end of the scale more salient and may thus facilitate learning, this question has not been rigorously tested (Lea1 \& Gaarder, 1971).

Biofeedback devices also differ with respect to input impedance characteristics (Schwitzgebel \& Rugh, 1975). It is generally agreed that the input impedance of a bioelectric amplifier should be at least 10 times greater than the electrode impedance (Geddes, 1972). When a high input impedance is employed, only a small current flows through the electrode impedance, and there is a minimal loss of voltage at the electrode-electrolyte interface. On the other hand, failure to have high input impedance with respect to electrode impedance can result in deterioration of the signal being measured (Peffer, 1979). When low input impedance devices are used to measure alpha activity, for example, what may be interpreted as increases in alpha activity may actually be the result of changes in the scalp-electrode impedance (Rugh, 1979). Some newer types of equipment use remote amplifiers that tend to reduce the impact of high electrode impedance on measurement of signal amplitude.

The sensitivity of the biofeedback equipment differs from device to device. It is important to know the resolution of the instrument that one is using (i.e., the minimum variation that can be accurately read). Otherwise, one may attempt to train an individual to make a change in a physiological response (e.g., lowering EMG from 5 microV to 2 microV) when the equipment is unable to discriminate a change that small.

Biofeedback instrumentation also differs in the choice of time constants provided. The time-constant dial sets the degree to which the feedback signal will follow rapid changes in the input signal. If a long time constant is used, only gross trends are presented, whereas if a short time constant is employed, the feedback will follow even slight fluctuations in input signal. Unfortunately, no parametric studies have been conducted to determine the optimum time constant for maximum training efficiency, and there is much variability on this parameter across studies (Rugh, 1979).

Clearly, it is important that the biofeedback user be aware of the technical characteristics of his/her instruments, since these instruments differ so greatly from manufacturer to manufacturer. It would be helpful if users would collect normative data from healthy individuals for each particular device and include information on types and specifications of apparatus used. It would also be helpful if users were more familiar with biofeedback electronics (e.g., Cohen, 1979) and instrumentation (e.g., Paskewitz, 1975; Peffer, 1979; Rugh, 1979; Yates, 1980). Furthermore, manufacturers of biofeedback instrumentation should begin to standardize equipment specifications and test procedures and to make specification sheets more informative and complete. A number of individuals have made recommendations for minimal standards in biofeedback instru- 
mentation specifications (e.g., Gaarder \& Montgomery, 1977; Girdano, 1976; Peffer, 1979). More attention should be given by the biofeedback user to the functional characteristics of his/her instruments (e.g., the bandwidth, signal-to-noise ratio, method of amplitude quantification, linearity of output signal, input impedance, sensitivity, and time constants).

\section{Biofeedback Protocol}

There are many choices that the user of biofeedback equipment has to make in the setting up and administration of the biofeedback protocol. As a result, biofeedback training protocols often differ greatly from one another. For example, the choice of feedback display is one that varies a great deal across investigators. Auditory feedback has been most commonly used, varying either in pitch or click rate as a function of change in physiological activity, and operating in derivative and inverted modes as well. There have been several types of visual displays employed in studies of biofeedback. Visual displays range from meters, to moving light bars, to videotaped feedback, to continuous oscilloscope displays, to digital displays. Investigations into the relative efficacy of these different feedback modalities have yielded mixed results (Alexander, French, \& Goodman, 1975; Blanchard \& Young, 1972; Budzynski \& Stoyva, 1973; Yates, 1980). In addition to whether or not one chooses auditory or visual displays of feedback, one must also select among eight possible combinations of the various "dimensions" of feedback display (Yates, 1980). Yates has described a taxonomy of feedback displays based on whether or not feedback is given within a trial or at the end of a trial, whether or not the feedback signal is proportional to the physiological measure or binary (i.e., the feedback is presented in an "on" or "off" mode, such that the feedback is displayed only when some physiological criterion is being met and is not displayed when the criterion is not being met), and whether or not the feedback is presented continuously or noncontinuously. In an examination of studies utilizing the various possible combinations of feedback displays (e.g., within trial, binary, noncontinuous feedback vs. within-trial, proportional, continuous feedback), Yates concluded that research to date on the feedback variable has been too unsystematic for clear-cut results to be obtained and that there may possibly be an interaction between types of feedback provided and the physiological response under investigation.

Another way in which biofeedback protocols vary has to do with the number and choice of sites being monitored. Between 1 and 16 channels are usually monitored, with feedback being provided for one physiological response, several sequential responses, or an integrated response pattern that takes many responses into account. Furthermore, the rationale for selection of particular sites may sometimes be arbitrary (e.g., EMG recording of the dorsal muscle of the forearm to assess physiological response to test anxiety; Thompson, Griebstein, \& Kuhlenschmidt, 1980), or based on unfounded assumptions (e.g., that reduction in frontalis EMG level is an indication of relaxation; Alexander, 1975). Numerous investigators have studied frontalis muscle EMG response as an indicator of anxiety or relaxation (Budzynski \& Stoyva, 1969; Canter, Kondo, \& Knotts, 1975; Raskin, Johnson, \& Rondestvedt, 1973; Townsend, House, \& Addario, 1975). However, "no actual experiment or experimental program has been undertaken to investigate the ability of a single EMG recording site (such as the forehead) to provide a valid, measurable, somatic indicator of general tension, relaxation or anxiety" (Alexander \& Smith, 1979, p. 120). In addition, there is little evidence that biofeedback-induced reductions in forehead muscle tension are accompanied by corresponding tension reduction in other muscles (Alexander, 1975; Shedivy \& Kleinman, 1977) or that there is even a transfer-of-training effect from one muscle site to another (Alexander \& Smith, 1979). Taken together, these findings suggest that as many sites as possible should be monitored, since one cannot assume equivalence of response across sites.

There is a diversity of procedures that may be followed within biofeedback training sessions. Length of session, frequency of sessions, number and timing of trials within a session, number and timing of rest periods within a session, length of habituation (baseline) period, characteristics of the environment, and instructions to trainees may all vary. While each of these may influence biofeedback outcome, there has been very little experimental investigation into the effects of these variables. McCanne and Sandman (1975) found that different results are obtained in heart rate biofeedback, depending on whether initial baseline or immediately preceding rest periods are used as the basis of feedback administration. Yates (1980) comments on the importance of conducting long baseline sessions of individuals in the resting state to guard against confounding influences created by "drift" effects (i.e., directional shifts in signal readings due to a variety of complex factors). "Drift" effects have been noted in studies of skin potential (e.g., Crider, Shapiro, \& Tursky, 1966), systolic and diastolic blood pressure (e.g., Glickstein, 1960), heart rate (e.g., Bouchard \& Granger, 1977), alpha waves (e.g., Crosson, Meinz, Laur, Williams, \& Andreychuk, 1977), peripheral temperature (e.g., Montgomery \& Williams, 1976), and EMG (e.g., Kinsman \& Staudenmayer, 1978).

It is clear that biofeedback training protocols vary greatly. Since the effects of these differences on outcome are relatively unknown, it may be important for future studies to examine protocol variables and to ultimately standardize biofeedback procedures.

\section{Data Acquisition and Analysis}

Because there are numerous ways in which biofeedback data can be collected and analyzed, studies often differ greatly in terms of procedures used. Some biofeed- 
back users record data by hand from fluctuating meter readings. This can be a laborious and frustrating task that is likely to be fraught with human error. Furthermore, decisions must be made concerning recording (e.g., one can record an instantaneous reading, or the approximate "average" value over $10 \mathrm{sec}$, or the range of values over $1 \mathrm{~min}$, etc.).

Some biofeedback users employ polygraphs. Unlike most methods of biofeedback data acquisition, polygraphs are capable of collecting very complete and continuous records of physiological responses. However, human data analysts are often required to interpret selected portions of the data, and the storage of accumulating paper output may be difficult. Sometimes polygraph outputs are fed directly into conventional laboratory minicomputers (e.g., PDP-11 and PDP-12) for analysis or are stored on FM tape for later data analysis. This type of computer use will be discussed later.

A relatively new way of collecting biofeedback information involves easily programmable multichannel biofeedback data acquisition devices designed to be easily integrated into most current biofeedback systems. This type of instrument partially automates the biofeedback system. One such data acquisition system, the Autogen 5600 (Autogenic Systems, Inc.), will be discussed later.

Another possible method of data compilation and analysis is through the use of "personal" or "hobbytype" microcomputers, which are inexpensive, powerful, and reliable. Unlike the data acquisition systems such as the autogenic systems, "personal" computers have to be specially integrated into the biofeedback system, and special software has to be developed.

A very recent development is the appearance of clinician-oriented customized microcomputers for automating biofeedback clinical and research work. These systems have much more flexibility than the data acquisition system mentioned above, but they are less expensive and more integrated as systems than generalpurpose laboratory-type computers are. In addition, these systems include easily accessed software that is customized for the needs of the behavioral medicine practitioner and the psychophysiologist. One such device, the Cyborg BioLab Computer System, is described later in this paper.

No matter which of the above data collection devices is employed (i.e., manual, polygraph, computer, data acquisition system), the biofeedback clinician/researcher has a variety of decisions to make regarding data analysis and data collection procedures, decisions that can introduce variability into the biofeedback procedure. Conflicting outcomes in reports of biofeedback effectiveness may be related to this variability. For example, while some investigators document changes in alpha wave production by scoring percent time in alpha above a specific amplitude, others integrate the area under the curve of the alpha signal. Hardt and Kamiya (1976), as well as Travis, Kondo, and Knot (1974), found that there is no linear relationship between these two methods of scoring alpha activity. While Hardt and Kamiya recommend the integrated measure (because it takes both amplitude and duration into account), many investigators employ the former measure alone. A similar situation exists in scoring EMG activity. Some investigators utilize measures that are integrated over several minutes' duration, others use shorter integration periods, and others do not integrate at all. Rugh (1979) recommends not integrating EMG signals over several minutes, particularly when recording from the frontalis area, since this may tend to average in the large periodic EMG activity associated with swallowing and movement. According to Rugh, "many of the dramatic reductions in EMG levels shown in the first few sessions of many published studies are possibly due to the subjects learning not to swallow during trial periods" (Rugh, 1979, p. 291).

Thus far, the discussion of variability in data collection procedures has dealt strictly with physiological measures. There is even more variability across research and clinical methodologies when one considers variables other than physiological ones. That is, some studies of biofeedback efficacy report outcome in terms of changes in one particular target physiological response (e.g., Budzynski \& Stoyva, 1969; Keefe, 1978; Keefe \& Gardner, 1979; Surwit \& Keefe, 1978). Other studies report outcome in terms of changes in several physiological measures (e.g., Alexander, 1975; Bakal \& Kaganov, 1977; Patewicz, 1976; Sargent, Green, \& Walters, 1972). In addition, others measure biofeedback outcome in terms of subjective self-report, behavioral measures, or psychological testing (e.g., Adler \& Adler, 1976; Bihldorff, King, \& Parnes, 1971; Chesney \& Shelton, 1976; Daniels, 1977; Lutker, 1971; Medina, Diamond, \& Franklin, 1976). And, finally, some utilize combinations of all of these measures (e.g., Budzynski et al., 1973; Cox, Freundlich, \& Meyer, 1975; Epstein, Able, Collins, Parker, \& Cinciripini, 1978; Feldman, Billingham, Kolotkin, \& Gots Bloch, Note 3). Depending on how one measures outcome, one is bound to find differences in terms of biofeedback effectiveness.

As in the discussions of biofeedback instrumentation and biofeedback protocols, it is apparent that much variability exists in techniques of biofeedback data acquisition and analysis. Unfortunately, unless some of this variability is reduced through standardization procedures, it is extremely difficult to compare studies on biofeedback effectiveness.

\section{ADVANTAGES OF COMPUTERS IN BIOFEEDBACK}

It is apparent that much variability exists in biofeedback instrumentation, biofeedback treatment protocols, techniques of biofeedback data acquisition, and types of biofeedback data analysis. One way to reduce some of this variability is to use computers. 
Computers introduce precision, accuracy, and reliability into the data acquisition process. The use of computers obviates the need for recording information from fluctuating meters or polygraph records and greatly reduces the human error in data collection. In a discussion of the applications of computers to a physiological psychology laboratory, Roemer $(1975$, p. 298) states that "indeed, most laboratories do not realize the percentage of human error actually involved in experimentation until a computer is used for comparison." While a high degree of precision and reliability is generally expected of a research laboratory, there is no reason to settle for less in the clinic. Certainly the task of treating clients is no less important than that of research. Furthermore, computers in the clinical setting enable clinicians to more accurately and reliably study patient populations, developing a data base within the most relevant context (Lang, 1980). The issue of adequately researching performance of patients in the context of the clinic becomes even more important when one considers the findings of Lang, Troyer, Twentyman, and Gatchel (1975) and Price and Tursky (1976) that patient groups cannot control autonomic activity as well as normals.

Unlike a human, a computer is capable of handling and recording large amounts of data rapidly. Whereas a human can focus on only limited subsets of clinical behaviors, computers can quickly monitor, analyze, and integrate multiple channels of information. As a result, the clinician/researcher is quickly and automatically provided a precise report of the trainee's progress, not only within that session but also compared with previous sessions and previous clients/subjects. Access to this information may enable the clinician/researcher to more effectively train his/her client or subject. Furthermore, with the computer doing all of the work of monitoring, analyzing, and integrating data within a session, the biofeedback operator has time to engage in therapeutic interactions with the trainee.

Another advantage of computers in biofeedback training is that computers can be used to create a structured biofeedback protocol. This means that all session parameters can be specified and defined a priori and then either held constant across and within individuals or systematically manipulated for experimental purposes. This might, for example, include the following parameters: total session length, frequency and timing of within-session trials and rest periods, amount of time spent in habituation (baseline), timing of data sampling and data analyses, timing and sensitivity of feedback, bandpass and threshold settings, and instructions to subjects.

A structured biofeedback protocol means that biofeedback training procedures can be easily replicated and, if desired, standardized across investigators. Of course, easily replicable, standardized procedures greatly reduce one source of variability described earlier. On the other hand, once a structured biofeedback protocol is created, it can be systematically manipulated for research purposes. One might, for example, be inter- ested in varying the length of training trials in order to determine if an optimum trial length exists for biofeedback training of a particular disorder. The computer can also be programmed to systematically vary the criteria for feedback signals to determine the level at which learning best occurs before frustration sets in. While Budzynski, Stoyva, and Adler (1970) chose an $80 \%$ criterion level (i.e., the gain was adjusted to maintain performance at or below a certain EMG amplitude 80\% of the time) in EMG training for tension headache, whether this is an optimal level for other types of patients must be investigated systematically. This type of question can be easily investigated in an automated biofeedback system.

Computers offer many advantages for data analysis and storage. Many simple on-line analyses are conducted instantaneously (e.g., means, standard deviations or lability indexes, maximum and minimum values, ranges, variances, and percent time at criterion). For more complex data analyses (e.g., curve fitting, trend analysis, correlation coefficients, multiple-regression analysis), information from bioelectric signals can be stored easily on tape and/or disks and analyzed at a later date. Visual displays of the data analysis (e.g., columns, graphs, and histograms) are also more easily produced by an automated system. Because of the computer's ability to monitor and analyze several sources of information at once, an automated biofeedback system can easily determine complex patterns of physiological responding. Furthermore, if behavioral, psychological, and historical data are also part of the computer's input, these types of data can be integrated with physiological data to provide the clinician/researcher with more global, better organized, and more accessible data about the individual. This enables the investigator/practitioner to identify unique pattems of physiological responding associated with particular individual characteristics and to ultimately tailor treatment programs more to the individual (Lang, 1980; Price \& Gatchel, 1979; Feldman et al, Note 3).

Another advantage of the computer is its "interactive" or "adaptive" capabilities (Lang, 1980; Roemer, 1975). This means that the computer can be programmed to be self-modifying according to the needs of the biofeedback trainee. A biofeedback protocol can, therefore, be continuously and automatically modified in accordance with previous responses of the trainee. This "adaptive" capacity makes the computer ideally suited for shaping responses according to the laws of operant conditioning. Subsequent stimulus presentations during a trial can be made dependent on an individual's immediate antecedent performance on some variable. In a matter of milliseconds, a contingency decision can be made and subsequent feedback stimuli presented. Both Lang (1980) and Roemer (1975) report on computer applications in the shaping of physiological responses.

In general, computers introduce flexibility into a biofeedback system. Several individuals can be run at 
once, with the various modalities being centrally monitored by one operator. If one wants to obtain nonresting baseline data, one can program the computers to administer a control task or a stressor to assess the subject's reaction to these stimuli. With computers, feedback can be made to correspond not only to instantaneous changes in physiological responses but also to trends in the total response pattern. Computers also have the capability of providing false feedback if needed in certain experimental designs. With computers, it is easier to administer feedback according to a set of complex contingencies utilizing several modalities at once (Paskewitz, 1975). For example, when a computer is used for EEG training, a power spectral analysis can provide the basis for a very sophisticated type of feedback representative of some combination of frequency and amplitude occurring in both hemispheres simultaneously (Fuller, 1978).

In summary, the advantages of utilizing computers in biofeedback are numerous. Not only can the computer be a helpful tool for biofeedback research, but it can also play an important role in the clinic.

\section{TYPES OF AUTOMATION}

Partial automation of the biofeedback system can be achieved with a multichannel biofeedback data acquisition device such as the Autogen 5600. Because this type of system is designed to be easily used by clinicians, it requires little specialized knowledge to program and is easily integrated into most current biofeedback systems. The Autogen 5600 enables automatic, comprehensive, asynchronous data compilation of up to 16 channels of analog and 4 channels of binary information. The system allows up to 16 patients to start and stop independently of each other, or, alternately, a variety of multiple-instrument combinations per individual trainee are possible (e.g., 4 patients, each training on four different feedback modalities). In this type of data acquisition system, physiological data are automatically and instantaneously analyzed and printed out. This includes a measure of the cumulative average value of a physiological response over a preselected period of time, a measure of percentage of time during which selected physiological criteria are being met, and a measure of standard deviation of a physiological response. The clinician/researcher may program the system to compute these measures over time intervals ranging from $1 \mathrm{sec}$ to $50 \mathrm{~h}$. Any session may be broken into 10 subintervals, so that not only are total computations provided for the entire session, but also up to 10 additional within-session computations are possible.

Instantaneous data analysis provides both the operator and the trainee with immediate knowledge of the trainee's progress that has taken place within a session, as well as between sessions. This immediate knowledge of results gives the trainee a type of feedback not previously available to persons undergoing biofeedback (i.e., numerical values representing statistically analyzed physiological activity in addition to the standard feedback signal). However, the effects of this type of feedback on training efficacy are unknown to date. An additional feature of this system is that it can create the type of structured biofeedback protocol described earlier, in which session parameters are either specified and held constant or systematically manipulated for research purposes. A disadvantage of this system is that it is only partially automated. Its primary goals are to collect data, to perform a few simple statistical analyses, and to create a structured protocol. It cannot, however, perform more complex data analyses, it is not "interactive" as described earlier (and, therefore, cannot be programmed to automatically shape responses), and it is generally not as flexible as the next two systems to be described.

A very recent development in biofeedback instrumentation is the appearance of clinician-oriented computers that are customized for biofeedback clinical and research work. One such device, the Cyborg Biolab Computer System, is a completely automated biofeedback system that has much more flexibility than the data acquisition system described above, yet it is less expensive and more integrated as a system than a generalpurpose laboratory computer. The system includes easily accessed software that is customized for the biofeedback clinician/researcher and that requires no previous computer or programming knowledge. Not only does the system administer a structured biofeedback protocol, conduct on-line analyses, and deliver feedback to trainees, but it also permits automatic shaping of trainee response by allowing the training criteria of one trial to be based on an individual's immediate antecedent performance on some variable. Furthermore, the Biolab software includes the following features: automated presentation, scoring, and interpretation of psychological tests; word processing for report writing; business and accounting options; and acceptance of traditional computer languages and publicly available software. The system replaces the polygraph with a continuous video graphics display that plots 250 points for each variable on the screen and saves the corresponding values for printout and for further data analysis. Up to 16 physiological input channels, as well as 8 analog, binary, or audio outputs, are available on this system. A maximum of eight persons may be trained simultaneously on this system. There is on-line data analysis on eight variables for each trial (consisting of the mean, standard deviation, variance, range, maximum and minimum values, first and last values, difference between first and last values, and percent threshold reached). At the end of each trial, information is stored on 8-in. floppy disks that can 
accommodate approximately 20-100 trials/disk. Elevenmegabyte hard storage disks are also available for more complete and longer term storage.

In contrast to the highly customized biofeedback computer systems described above is the traditional laboratory minicomputer that is useful not only for the biofeedback setting, but also for behavioral research in general (Castellan, 1975; B. Weiss, 1973). This type of computer is more flexible and versatile than customized computers and has additional core memory and storage capabilities as well. However, because it is a generalpurpose computer and not an integrated system, it is harder to implement a particular type of clinical or research protocol. Both the programming and the interfacing are more difficult to accomplish than in the above systems. In short, while this type of system has more versatility, it requires more computer experience and knowledge on the part of the users.

\section{RECENT RESEARCH IN AUTOMATED BIOFEEDBACK SYSTEMS}

In spite of the popularity of biofeedback research and the relatively widespread acceptance of computers, there are apparently very few published reports that deal explicitly with computer applications to biofeedback training. Roemer (1975) describes some recent applications of computers in psychophysiological research, including a research program by Schwartz and Fields at Harvard in which a PDP-11 is used for online biofeedback research. These researchers use the computer to experimentally manipulate the reinforcement contingencies of biofeedback (i.e., when the feedback stimulus is presented to the trainee). They specify to the computer the criteria for reinforcement and nonreinforcement of a physiological response, as well as the criteria for artifact. The feedback contingencies are such that su'ssequent feedback presentations during a trial are depend int on an individual's immediate antecedent performance on some particular physiological variable or on a combination of variables. Because of the rapidity with which the computer can make contingency decisions and present response-contingent feedback (several milliseconds), the computer is a useful aid for systematically shaping a biofeedback trainee's physiological responses.

A more detailed description (including hardware configuration, software description, flowcharts, and program source listings) of a similar computerized biofeedback training system for investigating the effects of response-contingent training strategies in EMG biofeedback-assisted relaxation therapy is presented by Pope and Gersten (1977). An on-line minicomputer (NOVA 2/10) monitors progress within the training session, compares the progress data with criteria set by the operator at the beginning of the session, and, on the basis of these comparisons, modifies the feedback path (by adjusting gain or sensitivity) and/or adjusts the within-session training sequence (by timing relaxation intervals, gating feedback signals, and selecting and presenting taped messages). Pope and Gersten describe in some detail a software-programmable "shaping" program that is designed to teach relaxation in a manner that is challenging, yet not frustrating. Pope and Gersten suggest using the computer for implementation of "shaping" strategies, as well as other training strategies (e.g., fading) that can be easily adapted to the needs of the particular individual being trained.

Lang's (1980) real-time computer system (LINC-8, PDP-12) is being utilized in research on biofeedbackassisted heart rate control. In addition to conducting data analyses, the computer is responsible for a variety of functions. These include presenting two kinds of visual feedback to subjects (e.g., successive moving lines that are proportional in length to the interpulse intervals of the EKG and the word "good" flashing on a screen whenever responses exceed a particular criterion), administering a shaping program to systematically train physiological responses in stepwise fashion, storing other types of physiological data (e.g., respiration rate, frontalis EMG, skin conductance level), administering control tasks to subjects (i.e., tasks against which performance in heart rate control can be assessed), and presenting instructions to subjects on a display screen. An extension of this automated biofeedback system is Lang's computer-assisted clinic for behavioral treatment, research, and training, which is currently under development. This elaborate computer-based operational system consists of a PDP-11/34 with a $64 \mathrm{~K}$-word memory and two RK05 disk drives, a DEC graphics unit, and several conversational terminals. Not only will the computer assist in particular behavioral treatments (e.g., biofeedback, systematic desensitization, programs for learning social skills), but the system will also be used for recordkeeping, supervising, test administration, diagnostic interviewing, the acquisition and analysis of behavioral observations, and on-line psychophysiological assessment. In this manner, the full range of client data will be systematized and easily accessible at a computer terminal in the clinic for clinical and research purposes.

In other applications of computers in biofeedback, Legewie and Probst (1969) used the computer (PDP-8L) to develop a new method of period-amplitude analysis of EEG activity. Beatty (1971) used a digital computer to conduct all phases of an experiment on the differential effects of response-contingent feedback vs. noncontingent feedback in the operant conditioning of alpha and beta wave activity. Konowe (Note 4) describes a multiparameter computerized approach to biofeed. back training that is being used for clinical research.

In summary, it seems that while computers have a lot to offer biofeedback researchers and clinicians, to 
date few automated biofeedback systems are in existence or are described in published reports.

\section{IMPLICATIONS}

The implications for the future of biofeedback are numerous. The many advantages of computers in biofeedback research and therapy have been enumerated in this paper. In addition, there is some indication that therapists, patients, and experimental subjects are becoming increasingly more comfortable interacting with computers (Biskin \& Kolotkin, 1977; Lang, 1980). These factors can be expected to lead to the more frequent use of computers in biofeedback. This increased usage of computers will help expand the field of behavior therapy, giving it a broad clinical base and introducing methods from the experimental analysis of behavior. With this expansion, future biofeedback clinician/ researchers may want to concern themselves with the following issues: tailoring of programs, branching techniques, modification of biofeedback protocols, and understanding the hows and whys of the biofeedback process.

The first issue for further consideration concerns the tailoring of programs. Given that there is a great deal of differential responsiveness of trainees to biofeedback, the researcher/clinician may want to determine which aspects of biofeedback work best under what circumstances and for which particular individuals or disorders (i.e., tailoring) (Kiesler, 1966; Price \& Gatchel, 1979). Not only may biofeedback be inappropriate for some types of problems and/or patients, but also certain aspects of the biofeedback protocol (length of time spent habituating to the environmental stimuli, type of feedback signal, etc.) may be more appropriate for some individuals than for others. Decisions about how to tailor programs to suit individual needs may be based on psychological factors (e.g., whether or not someone experiences intense anxiety). Or these may be based on behavioral factors (e.g., one's tendency to self-reward or to complain about pain) or physiological factors (e.g., symptomatology, lability of an organ system, tonic state of an organ system) (Feldman et al., Note 3 ).

Second, biofeedback researchers/clinicians may want to consider employing branching techniques in their training and research protocois. In general, branching techniques enable programs to be more individualized by the subject's participating in a unique subset of procedures that are determined by his/her previous responses. While branching techniques have been used by others in the area of ability measurement (D. J. Weiss, 1975), personality measurement (Kleinmuntz \& McLean, 1968), and diagnostic classification (Butcher, Clavelle, \& Hoffman, Note 5), they have largely been ignored in the area of biofeedback.
Another area for future consideration is the modification of biofeedback protocols in the hopes of creating more effective treatments. For example, Tursky (1979) proposes using proprioceptive or interoceptive feedback modalities (rather than auditory or visual) on the grounds that response consequences that are more consistent with naturally occurring afferent information may be more effective in producing sustained control of physiological systems. Another possibility is the addition of a monetary incentive (Lang \& Twentyman, 1976).

Finally, the future biofeedback researcher/clinician may want to concern himself/herself with developing a better understanding of how and why the biofeedback process works. A variety of possible explanations have been proposed in the biofeedback literature. Among these are that biofeedback works strictly according to the laws of operant conditioning, that it is an expensive placebo, and that it works as a distraction technique. Others propose that biofeedback involves the cognitive mediation of autonomic responses, that biofeedback teaches self-control coping skills, that it produces general relaxation, and that biofeedback enables one to see the relationship between physiological arousal and different emotional states. Future researchers can systematically investigate each of these possibilities. For example, Katkin and Goldband (1979) propose that researchers determine how biofeedback trainees respond to the various schedules of reinforcement, to delay of reinforcement, and to partial vs. continuous reinforcement in order to discover whether biofeedback is an active treatment that follows the laws of operant conditioning or whether biofeedback is more of a nonspecific treatment.

In summary, these and other issues provide the basis for future research and expanded clinical interventions, in part made possible by increased uses of computers in biofeedback.

\section{SUMMARY}

There has been a lack of replicability of findings across studies of biofeedback effectiveness. Although this may be due in part to experimental design issues or aspects of "person variables" in the process, problems may exist within the biofeedback system itself. This paper presents the issues involved with the biofeedback system.

Within the biofeedback system, it is apparent that one source of variability that contributes to differences in reports of biofeedback efficacy has to do with functional characteristics of the equipment. For example, differences in instruments' variations in bandwidth, methods of amplitude quantification, nature of output signals, and input impedance characteristics are discussed. Until manufacturers agree on a standardization 
program, the biofeedback clinician/researcher needs to be aware of the technical characteristics of his/her equipment, make these characteristics consistent across and within subjects, and report such information to facilitate replication. In addition, normative data from healthy individuals needs to be collected for different devices or systems.

A second source of variability is the biofeedback protocol. Research indicates mixed results concerning the type of feedback that is most effective. In addition, there is no consistency as to when feedback should occur (within trial or end of trial), whether the feedback signal should be binary or proportional to the physiological measure, and whether feedback should be continuous or noncontinuous. Other questions, such as number and location of physiological sites, number of sessions, length of sessions, and number of trials, need to be resolved. Research addressing these and other issues is needed, with the ultimate goal being the creation of standardized biofeedback protocols.

A third area for variability involves data acquisition and analysis. Manual recording procedures, polygraphs, computers, and data acquisition systems are all possible modes for data collection, and each has its own advantages and disadvantages. In addition, the biofeedback clinician/researcher has a variety of decisions to make regarding which particular data analysis to use and the type of data to collect.

The introduction of computers in biofeedback is advocated to help alleviate some of this variability. Several advantages of computers are presented. Among these is the greater precision, accuracy, and reliability in the data acquisition process. Human error would be reduced, large amounts of data could be recorded simultaneously, and data could be monitored, analyzed, and integrated within treatment sessions. In addition, computers can be used to create a structured biofeedback protocol. This means that all session parameters can be specified and defined a priori and then either held constant across and within individuals or systematically manipulated for experimental purposes. Greater flexibility can be gained when the computer self-modifies a biofeedback program to the needs of the trainee or when the computer monitors several individuals in various modalities simultaneously. These and other advantages are identified.

Several types of computer systems are evaluated (the Autogen 5600, the Cyborg Biolab Computer System, and the traditional laboratory minicomputer), and recent research in automated biofeedback systems is reviewed.

Finally, it is expected that the use of computers in biofeedback will help to expand the field of behavioral medicine, giving it a broad clinical base and introducing methods from the experimental analysis of behavior. Future biofeedback clinicians/researchers may want to concern themselves with the following issues: tailoring of programs, branching techniques, modification of biofeedback protocols, and understanding the hows and whys of the biofeedback process.

\section{REFERENCE NOTES}

1. Hart, J. T. Autocontrol of EEG alpha. Paper presented at the meeting of the Society of Psychophysiological Research, San Diego, October 1967.

2. Ancoli, S., \& Kamiya, J. Methodological issues in alpha feedback training. Paper presented at the meeting of the Biofeedback Society of America, Orlando, Florida, 1977.

3. Feldman, H. A., Billingham, K. A., Kolotkin, R, L., \& Gots Bloch, L. An innovative approach to the behavioral evaluation and management of headache. Paper presented to the Association for the Advancement of Behavior Therapy, San Francisco, December 1979.

4. Konowe, L. A multi-parameter computerized approach to biofeedback training. Paper presented at the meeting of the World Congress on Behavior Therapy, Jerusalem, Israel, July 1980.

5. Butcher, J., Clavelle, P., \& Hoffman, N. Clinical limitations of MMPI short forms and a strategy for developing a tailor-made MMPI short form. Paper presented at the 82nd Annual Convention of the American Psychological Association, New Orleans, September 1974.

\section{REFERENCES}

Adlen, C. S., \& Adlen, J. M. Biofeedback psychotherapy for the treatment of headache: A S-year follow up. Headache, 1976, 16, 189-191.

Alexander, A. B. An experimental test of assumptions relating to the use of electromyographic biofeedback as a general relaxation training technique. Psychophysiology, 1975, 12, 656-662.

Alexander, A. B., French, C. A., \& Goodman, N. A comparison of auditory and visual feedback in biofeedback assisted muscular relaxation training. Psychophysiology, 1975, 12, 119-123.

Alexander, A. B., \& Smith, D. C. Clinical applications of EMG biofeedback. In R. J. Gatchel \& K. P. Price (Eds.), Clinical applications of biofeedback: Appraisal and status. New York: Pergamon Press, 1979.

Bakal, D. A., \& Kaganov, J. A. Muscle contraction and migraine headache: Psychophysiologic comparison. Headache, $1977,17,208-215$.

BEATTY, J. Effects of initial alpha wave abundance and operant training procedures on occipital alpha and beta wave activity. Psychonomic Science, 1971, 23, 197-199.

Binldorf, J. P., King, S. H., \& Parnes, L. R. Psychological factors in headache. Headache, 1971, 11, 117-127.

Biskin, B. H., \& KolotKin, R. L. Effects of computerized administration on scores on the Minnesota Multiphasic Personality Inventory. Applied Psychological Measurement, 1977, 1, 543-549.

Blanchard, E. B., \& Epgtein, L. H. A biofeedback primer. Reading, Mass: Addison-Wesley, 1978.

Blanchard, E. B., \& Young, L. D. Relative efficacy of visual and auditory feedback for self-control of heart rate. Journal of General Psychology, 1972, 87, 195-202.

Blanchard, E. B., Young, L. D., \& Haynes, M. R. A single feedback system for the treatment of elevated blood pressure. Behavior Therapy, 1975, 6, 241-245.

Bouchard, M. A., \& Granger, L. The role of instructions 
versus instructions plus feedback in voluntary heart rate slowing. Psychophysiology, 1977, 14, 475-482.

Brener, J., Kleinman, R. A., \& Goesling, W. J. The effects of different exposures to augmented sensory feedback on the control of heart rate. Psychophysiology, 1969, 5, 510-516.

Budzynski, T. H., \& Stoyva, J. M. An instrument for producing deep muscle relaxation by means of analog information feedback. Journal of Applied Behavior Analysis, 1969, 2, 231 -237.

Budzynski, T. H., \& STorva, J. An electromyographic feedback technique for teaching voluntary relaxation of the masseter muscle. Journal of Dental Research, 1973, 52, 116-119.

Budzynsk1, T., Storva, J., \& AdLek, C. Feedback-induced muscle relaxation: Application to tension headache. Journal of Behavior Therapy and Experimental Psychiatry, 1970, 1, 205-211.

Budzynski, T. H., Storva, J. M., Adler, C. S., \& Mullaney, D. J. EMG biofeedback and tension headache: A controlled outcome study. Psychosomatic Medicine, 1973, 35, 484-496.

Canter, A., Kondo, C. Y., \& KnotT, J. R. A comparison of EMG feedback and progressive muscle relaxation training in anxiety neurosis. British Journal of Psychiatry, 1975, 127, 470-477.

Castellan, N. J. The modern minicomputer in laboratory automation. American Psychologist, 1975, 30, 205-211.

Chesney, M. A., \& Shelton, J. L. A comparison of muscle relaxation and electromyogram biofeedback treatments for muscle contraction headache. Journal of Behavior Therapy and Experimental Psychiatry, 1976, 7, 221-225.

Cohen, B. A. Basic biofeedback electronics for the clinician. In J. V. Basmajian (Eds.), Biofeedback: Principles and practice for clinicians. Baltimore, Md: Williams \& Wilkins, 1979.

Cox, D. J., Freundlich, A., \& Meyer, R. G. Differential effectiveness of EMG feedback, verbal relaxation instructions, and medication placebo with tension headaches. Journal of Consulting and Clinical Psychology, 1975, 43, 892-898.

Crider, A., Shapiro, D., \& Tursky, B. Reinforcement of spontaneous electrodermal activity. Journal of Comparative and Physiological Psychology, 1966, 61, 20-27.

Crosson, B., Meinz, R., Laur, E., Wilitams, D., \& ANDREYCHUK, T. EEG alpha training, hypnotic sussceptibility, and baseline techniques. International Journal of Clinical and Experimental Hypnosis, 1977, 25, 348-360.

Daniels, L. K. Treatment of migraine headache by hypnosis and behavior therapy: A case study. American Journal of Clinical Hypnosis, 1977, 19, 241-244.

Epstein, L. H., Abel, G. G., Collins, F., Parker, L., \& Cinciripini, P. M. The relationship between frontalis muscle activity and self-reports of headache pain. Behavior Research and Therapy, 1978, 16, 153-160.

Fuller, G. D. Current status of biofeedback in clinical practice. American Psychologist, 1978, 33, 39-48.

Gander, K. R., \& Montgomery, P. Clinical biofeedback: A procedural manual. Baltimore, Md: Williams \& Wilkins, 1977.

GedDes, L. A. Electrodes and the measurement of bioelectric events. New York: Wiley, 1972.

Gessel, A. H. Electromyographic biofeedback and tricyclic antidepressants in myofacial pain-dysfunction syndrome: Psychological predictors of outcome. Journal of the American Dental Association, 1975, 91, 1048-1052.

Grzonno, D. A. Buying biofeedback. In T. X. Barber, L. V. DiCara, J. Kamiya, N. E. Miller, D. Shapiro, \& J. Stoyva (Eds.), Biofeedback and self-control. Chicago: Aldine, 1976.

Guickste in, M. Temporal patterns of cardiovascular response. Archives of General Psychiatry, 1960, 2, 12-21.

HARDT, J. V., \& KamiYA, J. Conflicting results in EEG alpha feedback studies: Why amplitude integration should replace percent time. Biofeedback and self-regulation, 1976, 1, 63-75.

Hnatiow, M., \& Lang, P. J. Learned stabilization of cardiac rate. Psychophysiology, 1965, 1, 330-336.

Johnson, W. G., \& TuRiN, A. Biofeedback treatment of migraine headache: A systematic case study. Behavior Therapy, 1975, 6, 394-397.
Katkin, E. S., \& Goldband, S. The placebo effect and biofeedback. In R. J. Gatchel \& K. P. Price (Eds.), Clinical applications of biofeedback: Appraisal and status. New York: Pergamon Press, 1979.

KEEFE, F. J. Biofeedback vs. instructional control of skin temperature. Journal of Behavioral Medicine, 1978, 1, 383-390.

KEEFE, F. J., \& GARDNER, E. T. Learned control of skin temperature: Effects of short- and long-term biofeedback training. Behavior Therapy, 1979, 10, 202-210.

KIEsLER, D. J. Some myths of psychotherapy research and the search for a paradigm. Psychological Bulletin, 1966, 65, 110-136.

Kinsman, R. A., \& Staudenmayer, H. Baseline levels in muscle relaxation training. Biofeedback and Self-Regulation, 1978, 3, 97-104.

Kleinmuntz, B., \& Mclean, R. Diagnostic interviewing by digital computer. Behavioral Science, 1968, 13, 75-80.

LANG, P. J. Behavioral treatment and bio-behavioral assessment: Computer applications. In J. B. Sidowski, J. H. Johnson, \& T. A. Williams (Eds.), Technology in mental health care delivery systems. Norwood, N.J: Ablex, 1980.

Lang, P. J., Troyer, W. G., Twentyman, C. T., \& Gatchel, R. J. Differential effects of heart rate modification training on college students, older males, and patients with ischemic heart disease. Psychosomatic Medicine, 1975, 37, 429-446.

LANG, P. J., \& Twentyman, C. T. Learning to control heart rate: Effects of varying incentive and criterion of success on task performance. Psychophysiology, 1976, 13, 378-385.

Lazarus, R. S. A cognitive analysis of biofeedback control. In G. E. Schwartz \& J. Beatty (Eds.), Biofeedback theory and research. New York: Academic Press, 1977.

LeAF, W. B., \& GaAnder, K. R. A simplified electromyographic feedback apparatus for relaxation training. Journal of Behavior Therapy and Experimental Psychiatry, 1971, 2, 39-43.

Legewie, H., \& Probst, W. On-line analysis of EEG with a small computer (period-amplitude analysis). Electroencephalography and Clinical Neurophysiology, 1969, 27, 533-535.

LUTKER, E. Treatment of migraine headache by conditioned relaxation: A case study. Behavior Therapy, 1971, 2, 592-593.

McCanne, T. R., \& Sandman, C. A. Determinants of human operant heart rate conditioning: A systematic investigation of several methodological issues. Journal of Comparative and Physiological Psychology, 1975, 88, 609-618.

Medina, J. L., Diamond, S., \& Franklin, M. A. Biofeedback therapy for migraine. Headache, 1976, 16, 115-118.

Montgomery, L. D., \& Williams, B. A. Effect of ambient temperature on the thermal profile of the human forearm, hand, and fingers. Annals of Biomedical Engineering, 1976, 4, 209-219.

Nowlis, D. P., \& KamiYA, J. The control of electroencephalographic alpha rhythms through auditory feedback and the associated mental activity. Psychophysiology, 1970, 6, 476-484.

PAskewITZ, D. A. Biofeedback instrumentation: Soldering closed the loop. American Psychologist, 1975, 30, 371-378.

PATEwicz, E. P. "Cephalgic" spasm of head and neck muscles. Headache, 1976, 16, 261-266.

Peffer, K. E. Equipment needs for the psychotherapist. In J. V. Basmajian (Ed.), Biofeedback: Principles and practice for clinicians. Baltimore, Md: Williams \& Wilkins, 1979.

Phillips, C. The modification of tension headache pain using EMG biofeedback. Behavior Research and Therapy, 1977, 15, 119-129.

Pope, A. T., \& Gersten, C. D. Computer automation of biofeedback training. Behavior Research Methods \& Instrumentation, 1977, 9, 164-168.

PRICE, K. P. The application of behavior therapy to the treatment of psychosomatic disorders. Psychotherapy: Theory, Research and Practice, 1974, 11, 138-155.

Price, K. P., \& Gatchel, R. J. A perspective on elinical biofeedback. In R. J. Gatchel \& K. P. Price (Eds.), Clinical applications of biofeedback: Appraisal and status. New York: Pergamon Press, 1979. 
Price, K. P. \& Tursky, B. Vascular reactivity of migraineurs and nonmigraineurs: A comparison of responses to self-control procedures. Headache, 1976, 16, 210-217.

Raskin, M., Johnson, G., \& Rondestvedt, J. W. Chronic anxiety treated by feedback-induced muscle relaxation. Archives of General Psychiatry, 1973, 28, 263-267.

ROEMER, R. A. Some interactive computer applications in a physiological psychology laboratory. American Psychologist, 1975, 30, 295-298.

RUGH, J. D. Instrumentation in biofeedback. In R. J. Gatchel \& K. P. Price (Eds.), Clinical applications of biofeedback: Appraisal and status. New York: Pergamon Press, 1979.

RUGH, J. D., \& Schwitzogere, R. L. Variability in commercial electromyographic biofeedback devices. Behavior Research Methods \& Instrumentation, 1977, 9, 281-285.

Sargent, J., GreEn, E., \& Walters, D. The use of autogenic feedback training in a pilot study of migraine and tension headache. Headache, 1972, 12, 120-125.

Schedivy, D. I, \& KLEINman, K. M. Lack of correlation between frontalis EMG and either neck EMG or verbal ratings of tension. Psychophysiology, 1977, 14, 182-186.

Schwartz, G. E. Biofeedback as therapy: Some theoretical and practical issues. American Psychologist, 1973, 28, 666-673.

Schwitzoebel, R. L., \& RUGH, J. D. Of bread, circuses and alpha machines. American Psychologist, 1975, 30, 361-370.

Shapiro, D., Schwartz, G. E., Shimidman, S., Nelson, S., \& SiLvErman, S. Operant control of fear-related electrodermal responses in snake-phobic subjects. Psychophysiology, 1972, 9, 271.

Shapiro, D., Tursky, B., \& Schwartz, G. E. Differentiation of heart rate and systolic blood pressure in man by operant conditioning. Psychosomatic Medicine, 1970, 32, 417-423.
Silver, B. V., \& BlanchaRd, E. B. Biofeedback and relaxation training in the treatment of psychophysiological disorders: Or are the machines really necessary? Journal of Behavioral Medicine, 1978, 1, 217-239.

SURWIT, R. S. Biofeedback: A possible treatment for Raynaud's disease. In L. Bird (Ed.), Biofeedback: Behavioral medicine. New York: Grune \& Stratton, 1973.

Surwit, R. S., \& KeEfe, F. J. Frontalis EMG training: An electronic panacea? Behavior Therapy, 1978, 9, 779-792.

Thompson, J. G., Griebstein, M. G., \& Kuhlenschmidt, S. L. Effects of EMG biofeedback training in the prevention of academic underachievement. Journal of Counseling Psychology, 1980, 27, 97-106.

ToWnsEnd, R. E., House, J. F., \& AdDario, D. A comparison of biofeedback-mediated relaxation and group therapy in the treatment of chronic anxiety. American Journal of Psychiatry, $1975,132,598-601$.

Travis, T. A., Kondo, C. Y., \& KnotT, J. R. Parameters of eyes-closed alpha enhancement. Psychophysiology, 1974, 11, 674-681.

Tursxy, B. Biofeedback research methodology: Need for an effective change. In R. J. Gatchel \& K. P. Price (Eds.), Clinical applications of biofeedback: Appraisal and status. New York: Pergamon Press, 1979.

WEIss, B. Digital computers in the behavioral laboratory. New York: Appleton-Century-Crofts, 1973.

WEIss, D. J. (Ed.). Computerized adaptive trait measurement: Problems and prospects (Research Report 75-5). Minneapolis: University of Minnesota, Department of Psychology, November 1975. (NTIS No. AD-018675)

YATEs, A. J. Biofeedback and the modification of behavior. New York: Plenum, 1980. 